\title{
Longitudinal Spin Physics at COMPASS
}

\author{
E. M. Kabuss, ${ }^{1}$ \\ for the COMPASS collaboration
}

${ }^{1}$ Institut für Kernphysik, Universität Mainz, Germany, supported by the BMBF

January 23, 2012

\begin{abstract}
The spin structure of the nucleon is studied by the COMPASS collaboration using scattering of muons off polarised protons and deuterons. From the resulting asymmetries spin structure functions are extracted and the flavour decomposition of the quark contribution to the nucleon spin is studied. The gluon polarisation is investigated using the photon-gluon fusion process. Here, two main channels are investigated, open charm production and high $p_{T}$ hadron pair production. Also first measurements of unpolarised cross sections are reported.
\end{abstract}

keywords: Deep inelastic scattering, proton spin structure, gluon polarisation

\section{Introduction}

The structure of the nucleon is being studied in deep inelastic scattering (DIS) of leptons since more than forty years. Initially only experiments with unpolarised beams and targets were performed which studied the momentum distributions of quarks in the nucleon. When experiments with polarised beams and targets became available EMC made the puzzling finding that only little of the proton's spin is carried by quark spins [1]. At that time it was expected that quarks should carry at least $60 \%$ of the nucleon spin, mainly by valence quarks. The EMC result, often referred to as the "spin crisis", has led to a number of experiments with polarised lepton beams at CERN, DESY, SLAC and JLAB and with proton-proton collisions at RHIC studying the spin structure of the proton and the neutron in detail. All these experiments confirm the initial finding within the large uncertainty of the EMC results that only about $30 \%$ of the nucleon spin is carried by quarks. Other contributions obviously stem from gluon spins and orbital angular momenta of quarks and gluons.

To study these contributions new experiments are needed that not only focus on inclusive DIS but also include measurements of semi-inclusive (SIDIS) processes, i.e. measuring the produced hadrons in addition to the scattered lepton. One of the main players here is the COMPASS experiment at CERN with the possibility to study inclusive, semi-inclusive and exclusive reactions.

\section{The COMPASS experiment}

The COMPASS experiment [2] is situated in the north area of the CERN SPS at the unique M2 muon beamline. It delivers a highly polarised beam of $\mu^{+}$or $\mu^{-}$at energies between 100 and $200 \mathrm{GeV}$. Most 


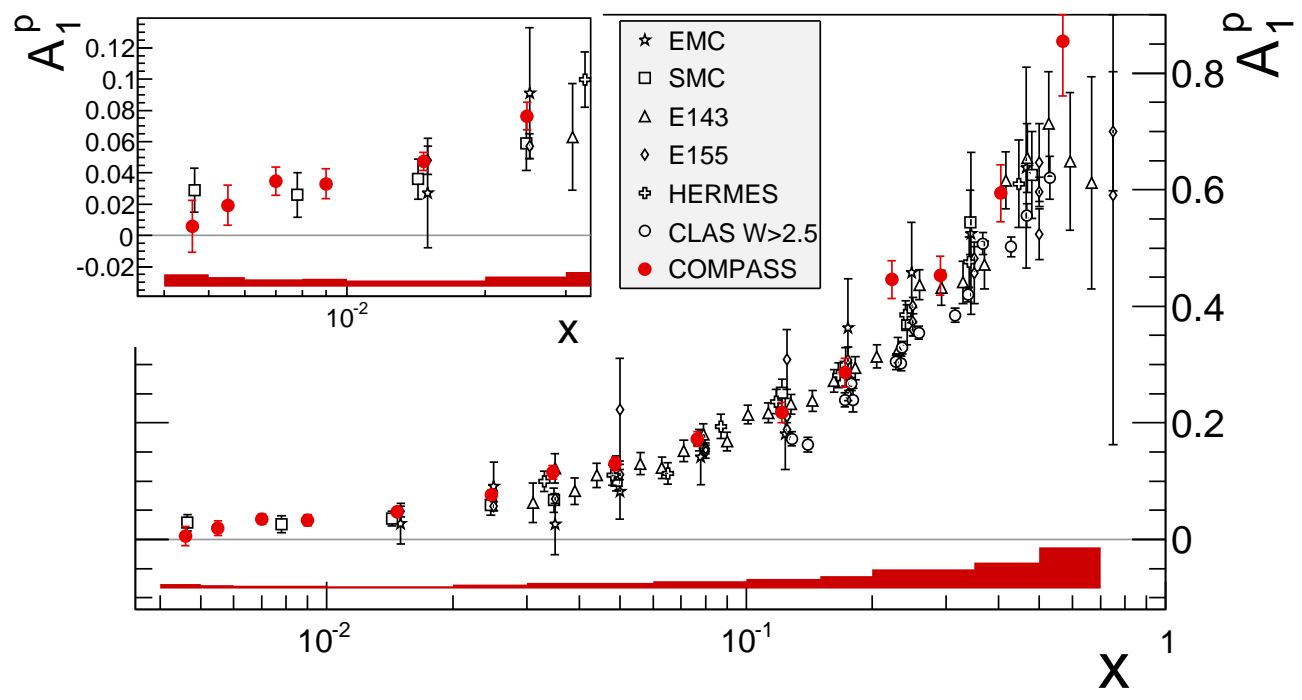

Figure 1: Compilation of results for $A_{1}^{p}$ versus $x$ [3]. Note the inprovement at low $x$ with the COMPASS data.

of the COMPASS data taking was done at $160 \mathrm{GeV}$ with an intensity of $4 \cdot 10^{7} \mu^{+} / \mathrm{s}$ at a spill length of $4.8 \mathrm{~s}(9.6 \mathrm{~s})$ for an SPS cycle of $16.8 \mathrm{~s}(40 \mathrm{~s})$ in 2002-2004 (2006-2011).

The heart of the experiment is the solid state polarised target housed in a large aperture superconducting solenoid with a field of $2.5 \mathrm{~T}$. The target material is polarised using dynamic nuclear polarisation and is cooled below $100 \mathrm{mK}$ with the help of a ${ }^{3} \mathrm{He} /{ }^{4} \mathrm{He}$ dilution refrigerator. The target polarisation is measured using $10 \mathrm{NMR}$ coils surrounding the target cells. The target arrangement is optimised for asymmetry measurements with two(three) cells simultaneously exposed to the muon beam. For this, the material was housed in two $60 \mathrm{~cm}$ long target cells in 2002-2004 and in three cells 30-60-30 cm long since 2006. Neighbouring cells are polarised in opposite directions. The target material was ${ }^{6} \mathrm{LiD}$ until 2006 and $\mathrm{NH}_{3}$ since then. A deuteron polarisation of about $50 \%$ was obtained for ${ }^{6} \mathrm{LiD}$ with a fraction of polarisable nucleons $f$ of 0.5 , while a proton polarisation of $90 \%$ is obtained for $\mathrm{NH}_{3}$ with $f$ about 0.15. The direction of the target polarisation is regularly reversed, three times per day until 2004 and only one time per day with the three cell set-up due to the much improved acceptance cancellation for asymmetry measurements.

The COMPASS spectrometer consists of a beam spectrometer in front of the target and a large acceptance double stage open dipole spectrometer behind the target. For each incoming muon the momentum and the direction are measured with high precision in scintillators and scintillating fibres with the help of a set of precise dipole magnets. After the target a variety of detector types are used to measure the tracks of the scattered muon and the forward going hadrons in two magnetic spectrometers, the first one measuring tracks with low momenta and large angle, while the second one picks fast, forward going particles. Muons are identified by their passage through concrete or iron absorbers, while electrons and hadrons are distinguished using ECAL/HCAL combinations in each stage. The first stage is also equipped with a RICH detector for $\pi / K / p$ separation.

\section{$3 \quad$ Asymmetry measurements}

In polarised DIS asymmetries are determined as a function of the negative four-momentum transfer squared $Q^{2}$ and the Bjorken variable $x$, which can be interpreted as the momentum fraction carried by the struck quark. For asymmetry determinations data from both(three) target cells from before and after a field reversal are combined to yield a physics observable [3]. By this method the acceptance which 


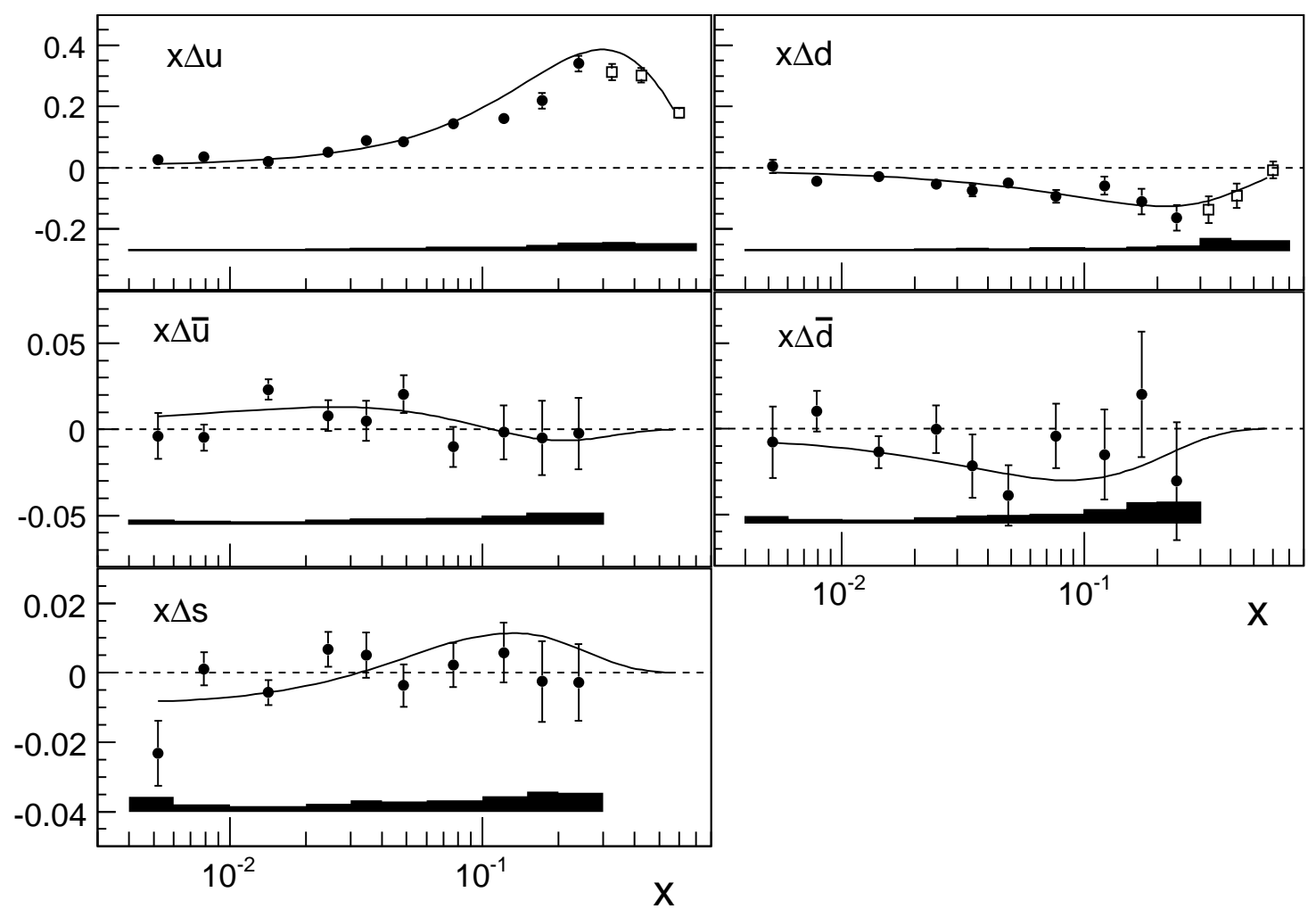

Figure 2: Quark helicity distributions as a function of $x$ [5]. The curves show NLO predictions of the DSSV fit [8].

is different for the different target cells and the luminosity which is different for the two measurements cancel. To achieve this cancellation, a very good stability of the spectrometer during the measurements has to be guaranteed. From the raw asymmetries the physics asymmetry $A_{\|}$is obtained by correcting for the target polarisation, the beam polarisation (80\%) and the fraction of polarised nucleons. As an example Fig. 1 shows the results from the 2007 data taking with polarised protons. Shown is the inclusive photon-nucleon asymmetry $A_{1}=A_{\|} / D$ in the DIS region for $Q^{2}>1(\mathrm{GeV} / c)^{2}$, where $D$ is the polarisation transfer from the lepton to the virtual photon. The data are compared to the world data on $A_{1}^{p}$ illustrating the large improvement at low $x$ with the COMPASS data. These data are used to determine the spin structure function $g_{1}^{p}$ and together with the deuteron results to test the fundamental Bjorken sum rule [3]. The data support the sum rule with an uncertainty of $7 \%$. From the first moment of the deuteron spin structure function $g_{1}^{d}$ the contribution of the quark helicity to the nucleon spin is determined to be $0.35 \pm 0.03$ (stat) \pm 0.05 (syst) where the contribution of a few $\%$ from the unmeasured region is determined using a next-to-leading order QCD analysis [4].

\section{Quark polarisation}

Adding to the inclusive asymmetry asymmetries for hadron production allows the contributions of the different quark flavours to the nucleon spin to be disentangled. COMPASS determined asymmetries for identified $\pi^{ \pm}$and $K^{ \pm}$from the deuteron and proton data [5] from 2002 to 2007. Here, only hadrons carrying more then $20 \%$ and less than $85 \%$ of the energy of the virtual photon are selected. The resulting polarised quark distributions are shown in Fig. 2. As no differences were observed between the $s$ and $\bar{s}$ polarisations, $s(x)=\bar{s}(x)$ is used in the final analysis. While a clear opposite polarisation signal is observed for $u$ and $d$ quarks, the sea quark polarisations are compatible with zero, including 
the strange quark polarisation. Here a negative polarisation of $5-10 \%$ is expected from NLO-QCD analyses. More data especially at low $x$ could shed more light on this discrepancy. Thus COMPASS took data in 2011 using a $200 \mathrm{GeV}$ beam, to extend the acceptance for $Q^{2}>1(\mathrm{GeV} / c)^{2}$ towards small $x$. Also, the uncertainty of the strange quark polarisation is sensitive to the fragmentation function used for strange quarks in the extraction. To improve the knowledge in the COMPASS kinematic range $K$ and $\pi$ multiplicities are being investigated for the 2004 deuteron data.

\section{Gluon polarisation}

There are two principal ways to study the gluon polarisation in DIS: an indirect method using NLOpQCD analyses and a more direct one making use of the photon-gluon fusion process. The analysis of the high statistics proton and deuteron $g_{1}$ data points to a small gluon polarisation, although the results depend on assumptions on the shape. Thus the systematic error of $\Delta g / g$ is large. More insight can be gained from the more direct measurements using photon-gluon fusion (PGF). Three analyses are performed at COMPASS focusing on different systematics aspects. The cleanest method makes use of open charm production. Due the negligible charm content of the nucleon and the suppression of charmed particles in the fragmentation process PGF is the dominant source of charmed mesons at COMPASS kinematics. For these events the charm quark mass yields the hard scale necessary for pQCD interpretation so that events with all $Q^{2}$ from the threshold onwards can be used. Open charm is tagged at COMPASS with the detection of $D^{0}$ and $\bar{D}^{0}$, reconstructed from their decay products, e.g. $K \pi$. Due to the large solid state target the $D_{0}$ decay vertices cannot be distinguished from the primary interaction vertices. Thus $D_{0}$ mesons can only be selected using the invariant mass distribution for identified $K \pi$ pairs. This selection is done with the help of a neural net which is trained on data with the help of a second sample containing only background events from wrong charge combinations. In the analysis not only $D$ decaying to $K \pi$ are selected but also four different decay channels of $D^{*} \rightarrow D^{0} \pi_{\text {slow }}$. The analysing power needed to extract the gluon polarisation is taken from MC simulations using the AROMA generator. This analysis is performed in LO and NLO pQCD [10].
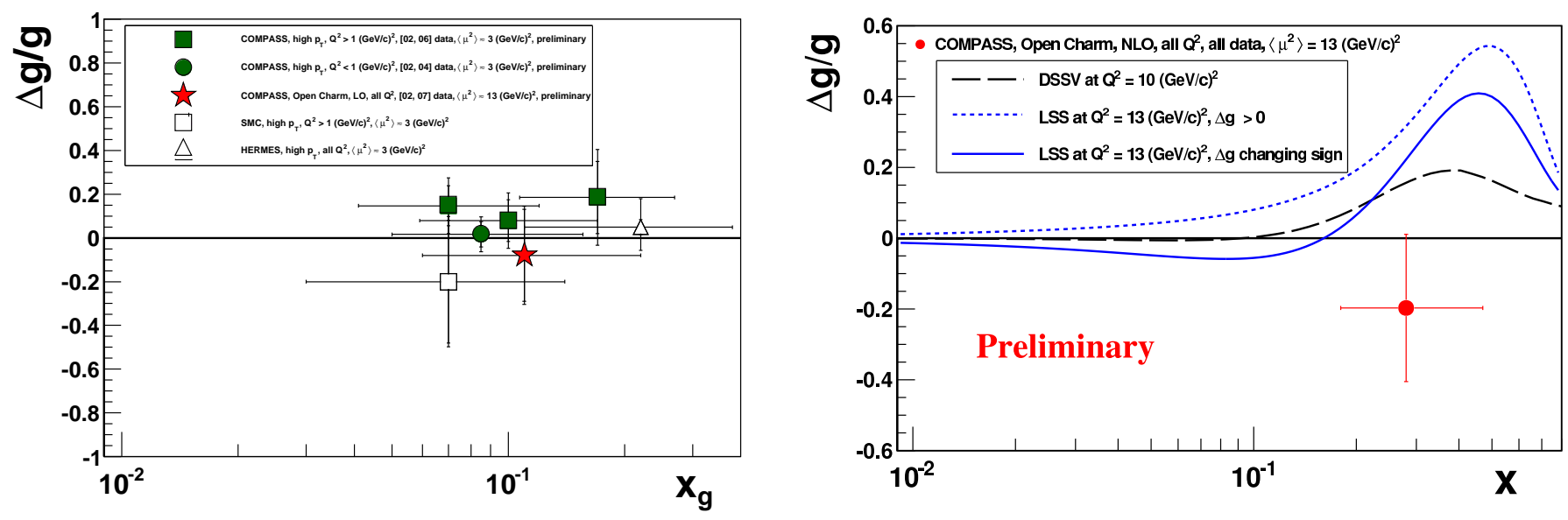

Figure 3: Compilation of gluon polarisation results at LO (left) and NLO (right). The curves display recent parametrisations of LSS [7] and DSSV [8] using the world DIS and SIDIS data.

In the second method hadron pairs with high transverse momenta $p_{T}$ are selected. In this case 
there are several contributions to the measured asymmetries, LO processes, QCD Compton and PGF processes. For low $Q^{2}$ events there are also resolved photon contributions. The analysis is done independently for data with $Q^{2}>1(\mathrm{GeV} / c)^{2}$ (DIS sample) and $Q^{2}<1(\mathrm{GeV} / c)^{2}$ (photoproduction sample). In the first case $Q^{2}$ can be used as the hard scale of the process, so that the reqirements on the hadron transverse momentum can be loosened, making up for the condsiderably lower statistics compared to the low $Q^{2}$ sample. Data selection employs a neural net which is trained on a Monte Carlo sample generated with LEPTO. Also the contributions from processes other than PGF have to be determined from Monte Carlo as well as the average kinematics and the analysing power. This analysis is done in LO pQCD. This method is employed to the 2002-2006 deuteron data. The obtained high statistics allows for the first time for a determination of the gluon polarisation in three bins of $x_{g}$ [11]. In the analysis of low $Q^{2}$ events the hard scale is given by the sum of $p_{T}^{2}$ of the two hadrons, requiring a stricter selection of the hadron pairs than for large $Q^{2}$ events. Up to now data from 2002 to 2004 have been analysed using LO pQCD as for the high $Q^{2}$ sample. In this analysis PYTHIA was used as event generator in the Monte Carlo simulation.

The results for the gluon polarisation are summarised in Fig. 3 (left) for the LO results and (right) for the open charm NLO result. As can be seen the measured gluon polarisation is compatible with 0 in the measured $x_{g}$ range. The NLO result is also compared to recent parametrisations from QCD analyses of all existing data.

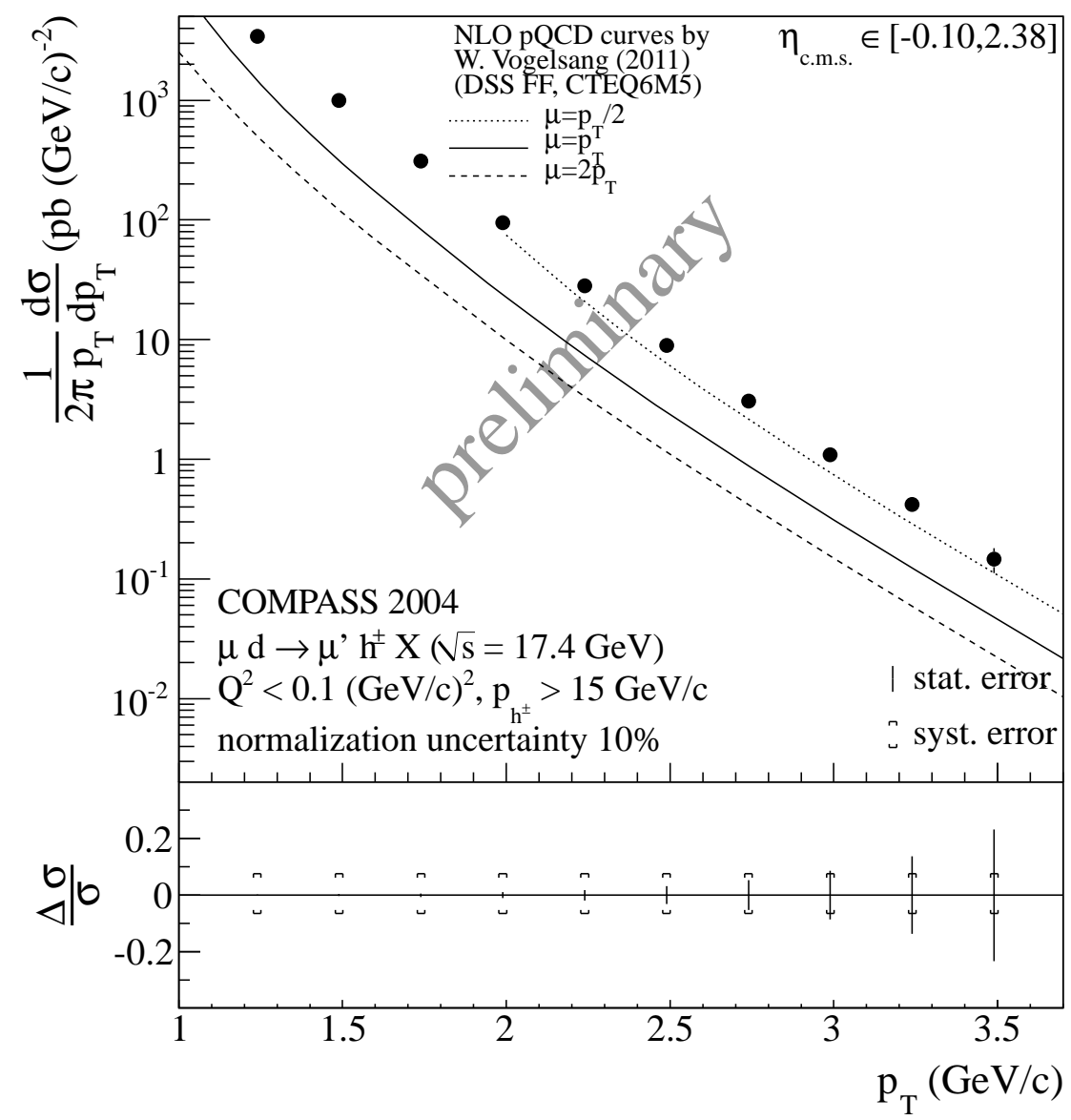

Figure 4: Cross section for charged hadron production as a function of $p_{T}$ for events with $Q^{2}<0.1(\mathrm{GeV} / c)^{2}$. The results are compared to NLO pQCD predictions, see ref. [9] and references therein. 
If one analyses high $p_{T}$ hadrons instead of hadron pairs at very low $Q^{2}$ the measured asymmetries can be compared with NLO pQCD predictions to extract the gluon polarisation. A first ingredient in such an analysis is the verification that the prediction agrees well with the unpolarised cross section. Thus first steps are taken to extract cross sections for high $p_{T}$ hadrons in the photoproduction region using events with $Q^{2}<0.1(\mathrm{GeV} / \mathrm{c})^{2}$. For such a measurement not only a good acceptance determination of the experimental set-up is mandatory but also a good measurement of the luminosity, a quantity usually not needed in asymmetry measurements. By now, it is possible to determine the luminosity with an uncertainty of $10 \%$ for part of the 2004 data. The extracted cross section for charged hadrons in shown in Fig.4 in comparison with the NLO pQCD prediction [12]. There is good agreement in shape but not in scale indicating that additional resummations have to be included in the predictions.

\section{Conclusion}

The COMPASS collboration has presented a wealth of new results on the longitudinal spin stucture. Here, results on quark and gluon polarisation were discussed in some detail. Future work will focus on improving the strange quark fragmentation function and single hadron asymmetries. In addition new data at a higher beam energy were taken in 2011. This will allow an improved determination of the proton spin structure function and the quark polarisation at small values of the quark momentum fraction.

\section{References}

[1] EMC, J. Ashman et al., Nucl. Phys. B 328 (1989) 1 .

[2] COMPASS, P. Abbon et al., Nucl. Instr. Meth. A 577 (2007) 455.

[3] COMPASS, M. Alkeseev et al., Phys. Lett. B 690 (2010) 466 .

[4] COMPASS, P. Abbon et al., Phys. Lett. B 647 (2007) 8 .

[5] COMPASS, M. Alkeseev et al., Phys. Lett. B 693 (2010) 227 .

[6] COMPASS, M. Alekseev et al., Phys. Lett. B 676 (2009) 31 .

[7] E. Leader, A.V. Sidorov and D.B. Stamenov, Phys. Rev. D 82 (2010) 114018.

[8] D. de Florian, R. Sassot, M. Stratmann and W. Vogelsang, Phys. Rev. D 80 (2009) 034040.

[9] B. Jäger, M. Stratmann and W. Vogelsang, Eur. Phys. Jour. C 44 (2005) 533.

[10] K. Kurek, Proc. of the 19th int. Workshop on Deep Inelastic Scattering, Newport News (2011), to be published.

[11] R. Silva, Proc. of the 19th int. Spin Physics Symposium, Jülich (2010), J. Phys. Conf. Ser. 295 (2011) 012067.

[12] Ch. Höppner, Proc. of the 14th int. Conference on Hadron Spectroscopy, Munich (2011), to be published. 